\title{
Current Status of Robot-Assisted Laparoscopic Partial Nephrectomy
}

\author{
Keng-Siang Png $\cdot$ Chandru P. Sundaram
}

Received: 10 September 2011 / Accepted: 23 September 2011/Published online: 7 October 2011

(C) Indian Association of Surgical Oncology 2011

\begin{abstract}
With increased incidence of diagnosis of small renal masses, partial nephrectomy has been preferred over radical nephrectomy as the surgical treatment of choice. The transition from open to laparoscopic partial nephrectomy had been challenging for many urologists. Robotic-assisted laparoscopic partial nephrectomy(RLPN) is increasingly used to facilitate this transition. In this review, we examine the recent technical advances and clinical outcomes in RLPN. Many series had successfully reported the feasibility of using the da Vinci Surgical (Intuitive Surgical Inc, Sunnyvale, CA) System in laparoscopic partial nephrectomy. Recent advances had focused on reducing risk of renal damage by shortening the warm ischaemia time. These techniques included unclamped excision, selective arterial clamping and improved renorrhaphy methods. Operative times and warm ischaemia times have also improved once the learning curve are overcome, which is less steep than conventional laparoscopy. With longer follow-up and more widespread experience, the outcome of RLPN could be favourable compared to conventional laparoscopy. Improving techniques had made this surgery a safe and efficacious treatment option for small renal masses.
\end{abstract}

Keywords Laparoscopy · Robotic surgery ·

Partial nephrectomy $\cdot$ Renal neoplasms

\section{Introduction}

With widespread imaging with computer tomography scans, small renal tumours are increasingly diagnosed [1-3]. In the

K.-S. Png $\cdot$ C. P. Sundaram $(\bowtie)$

Department of Urology, Indiana University School of Medicine,

535 N Barnhill Dr., Suite 420,

Indianapolis, IN 46202, USA

e-mail: sundaram@iupui.edu attempt to preserve renal function in patients with suspicious small renal masses, urologists had moved towards partial nephrectomy in their surgical management. It had also been established that the oncologic outcome of partial nephrectomy was comparable to radical nephrectomy [4-7]. The next step in partial nephrectomy was to transition from open to laparoscopic approach, with the attendant benefits of laparoscopic surgery, such as shorter convalescent time and hospital stay. However, many surgeons found conventional laparoscopic partial nephrectomy to be highly challenging because of intracorporeal excision and suturing while attempting to shorten ischemia time. The traditional laparoscopic approach was therefore not widely adopted. Over the last few years, robotic-assisted laparoscopic partial nephrectomy (RLPN) with 3-D vision and instruments with $6^{\circ}$ of freedom has allowed the surgery to be more feasible while decreasing warm ischemia time. In this review, we discuss the techniques used in RLPN and examine the reported clinical outcomes.

\section{Evolution of Robotics in Renal Surgery}

Laparoscopic nephrectomy had revolutionised renal surgery since the early 1990s [8]. The benefits of laparoscopic renal surgery such as shorter hospital stay, less pain, faster return to normal activities, better cosmesis were well-recognised [9]. In particular, obese patients were found to benefit from laparoscopic procedures with lower risk of postoperative wound and pulmonary complications [10, 11]. With the successful introduction of the Da Vinci Surgical system (Intuitive Surgical Inc, Sunnyvale, California) in radical prostatectomy, urologists had been keen to apply this advanced form of laparoscopy to renal surgery. Interestingly, in our experience, we had found that robotic-assisted laparoscopy was not found to be superior over conventional laparoscopy in radical nephrectomy [12]. As a result, most 
centers would still perform simple or radical nephrectomy through conventional laparoscopic means.

Partial nephrectomy, on the other hand, is well-suited for robotic assistance because of the requirement for instrument dexterity during the excision of the tumour and reconstruction of the collecting system and renal cortex. With conventional laparoscopic techniques, these tasks are difficult to achieve with the constraints of warm ischemia and limited degrees of motion with conventional instruments. The Da Vinci system had therefore been applied in this technically challenging operation.

\section{Operative Set-up}

The robotic set-up had been previously described by several centers [13-15]. With the patient in the $45^{\circ}$ modified flank position, the robot is positioned at the back of the patient with its arms straddling across the table over the patient. With the Da Vinci-S system, the fourth arm can be placed for additional retraction where required, such as during lateral retraction. The assistant performs several critical steps in this operation through additional one or two ports. These steps include application of hilar bulldog clamps and tumour localisation with laparoscopic ultrasound probe. Therefore, an experienced patient side assistant is essential. When compared to robotic-assisted laparoscopic prostatectomy, the biggest difference with RLPN is the wider surgical field. For this reason, care must be taken during port placements to avoid clashing between the robotic arms and the assistant instruments.

\section{Techniques to Lessen Ischaemic Damage or Ischemia Time}

Most of the recent advances made in RLPN were aimed at reducing the risk of nephron loss during warm ischemia. Intraarterial hypothermic perfusion was used in some early series of conventional laparoscopic partial nephrectomy [16, 17]. In this method, renal artery occlusion was performed using the intra-arterial catheter balloon with simultaneous iced saline infusion. Gettman et al. applied this technique to RLPN in 8 out of 13 patients of his initial reported series [15]. Later series mostly did away with renal arterial cooling and proceeded only with renal artery clamping [18].

In an attempt to shorten warm ischaemia time, early unclamping technique has been developed after placement of the first layer of medullary sutures. This technique, initially developed during the era of laparoscopic partial nephrectomy, resulting in halving of warm ischaemia time [19], has been used in RLPN, with median warm ischaemia time of $16 \mathrm{~min}$ in one report [20].
White et al. had reported no vascular clamping in eight patients with non-endophytic tumours less than $4 \mathrm{~cm}$ [21]. When compared to a contemporary group of RLPN patients with arterial clamping, operative time was significantly shorter while mean estimated blood loss was higher in the unclamped group. Operative outcomes and adverse events, including the need for a blood transfusion, were comparable in both groups.

Selective renal parenchymal clamping is another technique described to avoid arterial clamping. A special laparoscopic Simon clamp (Aesculap AG, Tuttlingen, Germany) is placed across the renal parenchyma immediately proximal to an exophytically-located tumour [22]. This was analogous to manual renal compression in open renal surgery to arrest bleeding during parenchymal breach. Developed for conventional laparoscopic partial nephrectomy, this technique has since been translated to the da Vinci platform, albeit in a small series [23].

Selective arterial clamping is a recent technique that had undergone evaluation in porcine models [24]. In this technique, only the segmental artery supplying the tumour was clamped. Results showed no significant rise in serum creatinine over a 7-day period. This promising technique would allow tumour excision and renorrhaphy to proceed without the constraint of main arterial clamping. Gill et al. had used and reported this technique in 15 patients [25]. Using combination of microdissection with selective ligation of branch renal artery/vein and hypotensive anaesthesia during deep tumour excision, bleeding was minimised with zero iscahemia. This series had recently been updated to 49 patients, with median resection time of $19 \mathrm{~min}$ (range 5 to $40 \mathrm{~min}$ ) and estimated blood loss $150 \mathrm{mls}$ (range 20$1500 \mathrm{mls})$ There were three high grade non-urologic complications and transfusions were required in 12 patients post-operatively [26].

To shorten the time taken for renorrhaphy and overall ischemia time, the sliding-clip technique for renorrhaphy had been described [27, 28]. Using Weck (Teleflex, Research Triangle Park, NC) Hem-o-lok clips placed at both ends of the suture, the console surgeon slid the clips to tighten the tension of the renorrhaphy to achieve a secure, haemostatic apposition of the cortical defect. This avoided knot-tying and could shortened hilar clamping time by up to 7 min [29].

Another technique to shorten renorrhaphy time is the use of barbed sutures. Barbed sutures, which were widely in plastic and gynaecologic surgery, have unidirectional etching to resist slippage through tissues and reduces the need for constant traction. After successful feasibility studies in porcine models [30]. The first clinical study was performed on 15 patients using the V-Loc 180 absorbable wound closure device (Covidien, Mansfield, MA) [31]. By comparing with preceeding 15 patients using 
conventional braided polyglactin suture (Vicryl, Ethicon, Johnson \& Johnson, Somerville, NJ), the authors had shown significant shorter warm ischaemia time in the VLoc group (18.5 vs $24.7 \mathrm{~min}$ ). With the Vicryl group, additional clips had to be applied regularly during renorrhaphy to prevent slipping. This was not required in the VLoc group.

\section{Technique of Tumour Excision and Renorrhaphy at the Indiana University}

At Indiana University, we apply laparoscopic bulldog clamps to the artery and the vein separately prior to excision of the tumour. Exposure of the hilar vessels is aided by using two short 5 -inch vessel loops to encircle the artery and vein. These vessel loops are clipped with a polymer clip. Traction on these loops can then facilitate the placement of the bulldog clamps. In cases of difficult hilar dissection, we would use a laparoscopic Satinsky clamp applied by the assistant to mass-clamp the hilum. One must be careful to avoid external collisions in this case. After identifying the location of the tumour with laparoscopic ultrasound, the renal capsule around the tumour is scored to provide a line by which excision of the tumour can follow. Excision of the tumour is performed with curved scissors initially directed away from the tumour. Retraction is provided by the fenestrated bipolar forceps or PK dissector on the left arm and the assistant's suction-irrigator. A biopsy of the resection bed is taken and sent for frozen section analysis. Initial haemostasis is obtained using argon-beam coagulator directed towards the cortex, avoiding the more central area to avoid thermal damage to the collecting system.

To repair the defect, we prefer a two-layer closure. The deeper layer consisting of the collecting system, blood vessels and deep cortex is closed with a $2 / 0$ polyglactin or barbed absorbable suture attached at the end with a $10 \mathrm{~mm}$ polymer clip and a Lapra-Ty clip (Ethicon Endosurgery, Cincinnati, Ohio). Deep bites within this layer are avoided as this could injure deeper blood vessels, contributing to formation of pseudoaneurysms and arteriovenous fistulas. After running the entire resection bed, the suture is passed out through the cortex and anchored with a $10 \mathrm{~mm}$ polymer clip. It is important to ensure that the non-absorbable clips are on the renal cortical surface and not adjacent to the collecting system, to prevent migration into the collecting system. For the outer cortical layer repair, we prefer using a single-arm polydioxanone 0 barbed suture in a horizontal mattress configuration. After each pass through the cortex, the closure is reinforced with a polymer clip and slid down to the desired tension. The clamps are removed after completion of the outer layer closure. The ends of both inner and outer layer sutures are reinforced with Lapra-Ty clips. A gelatin-thrombin mixture is added to the closure.

\section{Clinical Outcomes}

The first reported series of RLPN consisted of small number of patients and the results merely showed feasibility of the operation $[13,15]$. Despite the perceived advantages of wristed instruments, there was no demonstrable benefit over conventional laparoscopic partial nephrectomy [18].

In more recent multi-center series, the clinical outcomes are more promising. The largest published comparative series between 118 conventional laparoscopic partial nephrectomy and 129 RLPN cases had shown no difference in operative times [32]. Intra-operative blood loss was less in the RPLN group (155 mls vs $196 \mathrm{mls}$ ), as was length of hospital stay (2.4 vs 2.7 days), though this was not clinically significant. Warm ischemic times were also shorter in the RPLN group(19.7 vs $28.4 \mathrm{~min}$ ). Postoperative complications were similar in both groups, as was the positive margin rate. In another published large comparative series from a single center, RPLN again showed shorter operative time (140 vs $156 \mathrm{~min}$ ) and shorter warm ischemia time (19 vs $25 \mathrm{~min}$ ) with no difference in estimated blood loss and positive margin rate [33].

In the largest single-arm series of RLPN published to date with 189 patients, the mean operative time was $210 \mathrm{~min}$ while mean ischemic time was $23.9 \mathrm{~min}$. The positive margin rate was found in $3.8 \%$ of pathological specimens, of which none exhibited disease recurrence on follow-up [34].

At the AUA 2011 Annual Meeting, a collection of 1035 cases of RLPN was presented from 23 institutions. Warm ischaemia time was $24.7 \mathrm{~min}$ (range 6 to $77 \mathrm{~min}$ ), estimated blood loss was $216 \mathrm{ml}$ (range 5 to $2,800 \mathrm{mls}$ ) and $96 \%$ exhibited negative pathological surgical margins [35]. This series had shown that RLPN could consistently be performed safely across various institutions.

The data from these series are summarized in Table 1.

\section{Conclusion}

Randomised studies between conventional laparoscopic partial nephrectomy and RLPN will be difficult to conduct and may never materialise. In its absence, existing comparative studies had shown great promise with RLPN in terms of clinical and early oncologic outcomes. With more centers performing RLPN and longer term follow-up, RLPN would continue to mature in its role in the management of renal tumors. 







\section{References}

1. Chow WH, Devesa SS, Warren JL, Fraumeni JF Jr (1999) Rising incidence of renal cell cancer in the United States. JAMA 281 (17):1628-1631

2. Hollingsworth JM, Miller DC, Daignault S, Hollenbeck BK (2006) Rising incidence of small renal masses: a need to reassess treatment effect. J Natl Cancer Inst 98(18):1331-1334

3. Jayson M, Sanders H (1998) Increased incidence of serendipitously discovered renal cell carcinoma. Urology 51:203-205

4. Marszalek M, Meixl H, Polajnar M, Rauchenwald M, Jeschke K, Madersbacher S (2009) Laparoscopic and open partial nephrectomy: a matched-pair comparison of 200 patients. Eur Urol 55 (5): $1171-1178$

5. Peycelon M, Hupertan V, Comperat E, Renard-Penna R, Vaessen C, Conort P, Bitker MO, Chartier-Kastler E, Richard F, Rouprêt M (2009) Long-term outcomes after nephron sparing surgery for renal cell carcinoma larger than $4 \mathrm{~cm}$. J Urol 181(1):35-41

6. Gill IS, Kavoussi LR, Lane BR, Blute ML, Babineau D, Colombo JR Jr, Frank I, Permpongkosol S, Weight CJ, Kaouk JH, Kattan MW, Novick AC (2007) Comparison of 1,800 laparoscopic and open partial nephrectomies for single renal tumors. J Urol 178 (1):41-46

7. Delakas D, Karyotis I, Daskalopoulos G, Terhorst B, Lymberopoulos S, Cranidis A (2002) Nephron-sparing surgery for localized renal cell carcinoma with a normal contralateral kidney: a European threecenter experience. Urology 60(6):998-1002

8. Clayman RV, Kavoussi LR, Soper NJ et al (1991) Laparoscopic nephrectomy. N Engl J Med 324:1370

9. Burgess NA, Koo BC, Calvert RC, Hindmarsh A, Donaldson PJ, Rhodes M (2007) Randomized trial of laparoscopic V open nephrectomy. J Endourol 21(6):610-613

10. Hedican SP, Moon TD, Lowry PS, Nakada SY (2004) Handassisted laparoscopic renal surgery in the morbidly and profoundly obese. J Endourol 18(3):241-244

11. Kapoor A, Nassir A, Chew B, Gillis A, Luke P, Whelan P (2004) Comparison of laparoscopic radical renal surgery in morbidly obese and non-obese patients. J Endourol 18(7):657-660

12. Boger M, Lucas SM, Popp SC, Gardner TA, Sundaram CP (2010) Comparison of robot-assisted nephrectomy with laparoscopic and hand-assisted laparoscopic nephrectomy. JSLS 14(3):374-380

13. Phillips CK, Taneja SS, Stifelman MD (2005) Robot-assisted laparoscopic partial nephrectomy: the NYU technique. J Endourol 19(4):441-445

14. Patel MN, Bhandari M, Menon M, Rogers CG (2009) Roboticassisted partial nephrectomy. BJU Int 103(9):1296-1311

15. Gettman MT, Blute ML, Chow GK, Neururer R, Bartsch G, Peschel R (2004) Robotic-assisted laparoscopic partial nephrectomy: technique and initial clinical experience with DaVinci robotic system. Urology 64(5):914-918

16. Marberger M, Georgi M, Guenther R, Hohenfellner R (1978) Simultaneous balloon occlusion of the renal artery and hypothermic perfusion in in situ surgery of the kidney. J Urol 119(4):463467

17. Janetschek G, Abdelmaksoud A, Bagheri F, Al-Zahrani H, Leeb K, Gschwendtner M (2004) Laparoscopic partial nephrectomy in cold ischemia: renal artery perfusion. J Urol 171(1):68-71
18. Caruso RP, Phillips CK, Kau E, Taneja SS, Stifelman MD (2006) Robot assisted laparoscopic partial nephrectomy: initial experience. J Urol 176(1):36-39

19. Nguyen MM, Gill IS (2008) Halving ischemia time during laparoscopic partial nephrectomy. J Urol 179(2):627-632

20. San Francisco IF, Sweeney MC, Wagner AA (2011) Robotassisted partial nephrectomy: early unclamping technique. J Endourol 25(2):305-308

21. White WM, Goel RK, Haber GP, Kaouk JH (2010) Robotic partial nephrectomy without renal hilar occlusion. BJU Int 105 (11): $1580-1584$

22. Simon J, Bartsch G, Finter F et al (2008) Laparoscopic partial nephrectomy with selective control of the renal parenchyma: initial experience with a novel laparoscopic clamp. BJU Int 103:8805-8808

23. Viprakasit DP, Altamar HO, Miller NL, Herrell SD (2010) Selective renal parenchymal clamping in robotic partial nephrectomy: initial experience. Urology 76(3):750-753

24. Benway BM, Baca G, Bhayani SB et al (2009) Selective versus nonselective arterial clamping during laparoscopic partial nephrectomy: impact upon renal function in the setting of a solitary kidney in a porcine model. J Endourol 23(7):1127-1133

25. Gill IS, Eisenberg MS, Aron M et al (2011) "Zero ischemia" partial nephrectomy: novel laparoscopic and robotic technique. Eur Urol 59(1):128-134

26. Eisenberg M, Desai M, Berger A et al (2011) 1078 "Zero-ischemia" partial nephrectomy: Novel laparoscopic and robotic technique. J Urol 185((4) Suppl):e433-e434

27. Cabello JM, Benway BM, Bhayani SB (2009) Robotic-assisted partial nephrectomy: surgical technique using a 3-arm approach and sliding-clip renorrhaphy. Int Braz J Urol 35(2):199-203

28. Benway BM, Cabello JM, Figenshau RS, Bhayani SB (2010) Sliding-clip renorrhaphy provides superior closing tension during robot-assisted partial nephrectomy. J Endourol 24(4):605-608

29. Benway BM, Wang AJ, Cabello JM, Bhayani SB (2009) Robotic partial nephrectomy with sliding-clip renorrhaphy: technique and outcomes. Eur Urol 55(3):592-599

30. Shikanov S, Wille M, Large M, Lifshitz DA, Zorn KC, Shalhav AL, Eggener SE (2009) Knotless closure of the collecting system and renal parenchyma with a novel barbed suture during laparoscopic porcine partial nephrectomy. J Endourol 23(7):1157-1160

31. Sammon J, Petros F, Sukumar S, Bhandari A, Kaul S, Menon M, Rogers C (2011) Barbed suture for renorrhaphy during robotassisted partial nephrectomy. J Endourol 25(3):529-533

32. Benway BM, Bhayani SB, Rogers CG et al (2009) Robot assisted partial nephrectomy versus laparoscopic partial nephrectomy for renal tumors: a multi-institutional analysis of perioperative outcomes. J Urol 182(3):866-872

33. Wang AJ, Bhayani SB (2009) Robotic partial nephrectomy versuslaparoscopic partial nephrectomy for renal cell carcinoma: single-surgeon analysis of $>100$ consecutive procedures. Urology 73(2):306-310

34. Benway BM, Bhayani SB, Rogers CG et al (2010) Eur Urol 2010. Robot-assisted partial nephrectomy: an international experience. Eur Urol 57(5):815-820

35. Munver R, Yates J, Sundaram C et al (2011) Robot-assisted partial nephrectomy in 1035 consecutive cases: a multi-institutional experience. J Urol 185((4)Suppl):e743-e744 